\title{
NUMERICAL SIMULATION OF THE FLOW IN WIRE-WRAPPED PIN BUNDLES: EFFECT OF PIN-WIRE CONTACT MODELING
}

\author{
E. Merzari, W. D. Pointer, J. G. Smith \\ Nuclear Engineering Division, Argonne National Laboratory, 9700 S. Cass Avenue, Argonne, IL \\ 60439,.USA,emerzari@anl.gov,+16302526637 \\ P. Fischer
}

Mathematics and Computer Science Division, Argonne National Laboratory, 9700 S. Cass Avenue, Argonne, IL 60439, USA

\begin{abstract}
The rapid advancement of numerical techniques and the availability of increasingly powerful supercomputers recently enabled scientists to use large eddy simulation (LES) to simulate numerically the flow in a full subassembly composed of wire-wrapped pins. Because of the extreme computational cost of such simulation, it was not possible to conduct a sensitivity case on the pin-wire interface modeling. Since such calculations are likely to be extended to conjugate heat transfer, however, a sensitivity study is necessary to assess the reliability of the numerical results. It is well known that conjugate heat-transfer results are often strongly influenced by near-wall modeling. The objective of the present work is to investigate the effect of pin-wire contact modeling from the point of view of both the hydraulics and the heat transfer characteristics. In particular, the focus is on the prediction of the hot spot in conjugate heat-transfer calculations. The primary test case is the simplified geometry recently proposed by Ranjan et al., which consists of a simple channel flow with a wire embedded in one of the walls. After reproducing the results using the LES code Nek5000, we examined several other choices for the wire-pin interface modeling, including the introduction of a nominal gap between the wire and wall. The results shed light on the sensitivity of LES calculation results to the modeling of the interface region between wires and pins.
\end{abstract}

\section{INTRODUCTION}

The study of conjugate heat transfer is needed for accurately simulating the flow and temperature fields of a nuclear reactor core. With computational fluid dynamics-based techniques and the use of supercomputing platforms, one can now achieve a remarkable degree of accuracy for the most relevant turbulence factors, such as the average velocity field, the averaged temperature distribution, and the stresses inside the fluid region. The coupling of the fluid dynamics problem with the conduction equation solved inside the fuel pin is less straightforward, however. In particular, the choices made in modeling the contact between the fuel pin and other structural components (such as spacers or wires in the case of sodium-cooled fast reactors) might lead to sensitive perturbations on the prediction of the hot spot (i.e., peak temperature in the solid) or other thermodynamic and hydrodynamic properties, since such details have effects on other geometries ( $\mathrm{Qu}$ and Mudawar, 2002).

For instance, Pointer et al. (2009) recently used large eddy simulaton (LES) to simulate numerically the flow in a full subassembly composed of wire-wrapped pins. Because of the extreme computational cost of such simulations, it was not possible to conduct a sensitivity study of the pin-wire interface modeling. Since such calculations are likely to be extended to conjugate heat transfer, however, a sensitivity study is necessary in order to assess the reliability of the numerical results.

In fact, the wire introduces a higher resistance to conduction in the radial direction which, combined to the high conductivity of sodium, might lead to a significant localized hot spot. The presence of local stagnation points might also affect the temperature distribution in the fluid (Tang et al., 1978). The 
way the interface is modeled has the potential to affect the value of the hot spot, since it would affect the hydrodynamics and the local heat transfer. Moreover, in some instances, the wire might be in contact with the pin, depending on design and operational conditions. Thus, to the uncertainty due to numerical modeling one should add uncertainty due to the variability of the physical conditions.

The main purpose of the present work is to shed some light on how pin-wire contact modeling affects the hot-spot prediction in a simplified geometry. Arguably, such a study would be problematic in an actual core because the hot-spot position and value are determined essentially by the axial position (due to the nonuniformity of the power distribution; see Khakim et al., 2009, and Baglietto and Ninokata, 2006) and it would be nearly impossible to separate the small but potentially significant effect due to the modeling of the wire or other structural components.

The primary test case is the geometry proposed by Ranjan et al. (2010), which consists of a simple channel flow with a wire embedded in one of the walls. Ranjan et al. provided detailed and extremely accurate (i.e., obtained with highly resolved direct numerical simulation techniques) distributions for the velocity field and other turbulent statistics, thus allowing us to fully verify and validate our calculations.

After reproducing the results using the LES code Nek5000 (Fischer et al., 2008), we examined the conjugate heat-transfer problem (which was not studied by Ranjan et al., 2010). We also considered an additional modeling option for the wire: the introduction of a nominal gap between the wire and wall. We then used the commercial code STAR-CCM+ in RANS mode to study the same problem, with the double purpose of verifying the results obtained and testing the capability of RANS-based methods to achieve accurate results for the present case.

\section{METHODOLOGY}

In this section we describe the two wall-wire contact models used. We present the mathematical model and the relevant parameters chose, and we discuss the methodology used for the simulations.

\subsection{Problem description}

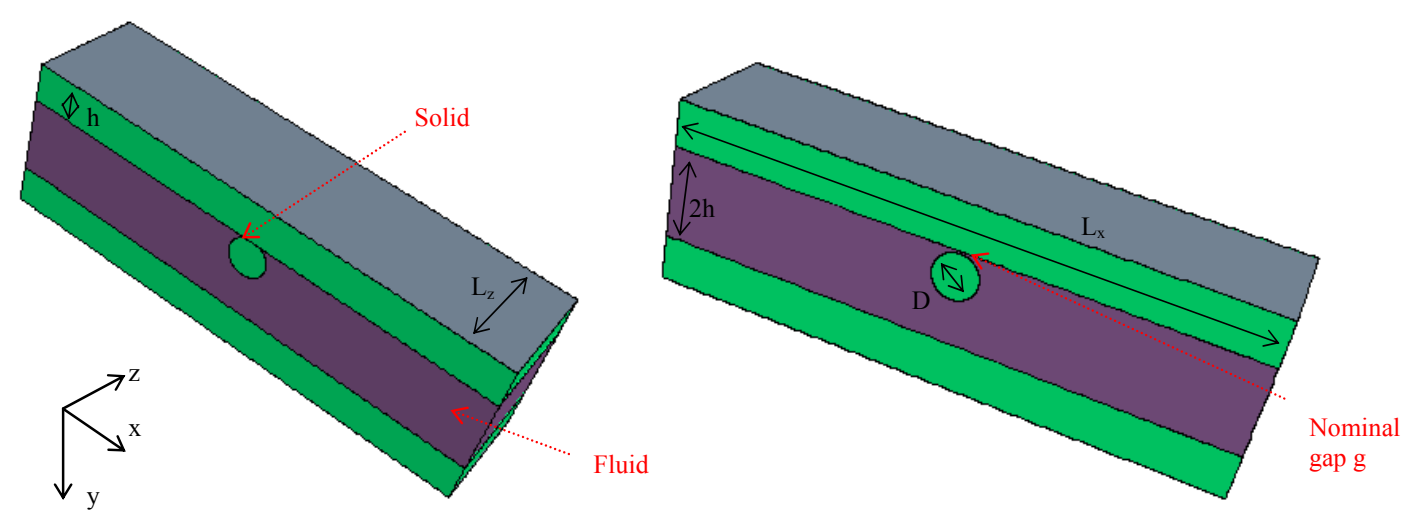

Fig. 1. Three-dimensional representation of the case with (left) contact between wire and wall and (right) no contact between meshes.

The direct numerical simulation (DNS) calculation of Ranjan et al. (2010) was selected for its generality, its high resolution (it was computing over 100,000,000 collocation points), and its relatively low Reynolds number. The geometry investigated in that paper corresponds to a wire 
immersed in a channel flow with both streamwise (direction $\mathrm{z}$ ) and spanwise (direction $\mathrm{x}$ ) periodic boundary conditions (the boundary conditions are identical for Nek5000 and STAR-CCM+). The geometry is shown in further detail in Fig. 1.

In the present work, while keeping the same outline for the geometry, we explore two wall-wire contact models. In the first case we keep the same contact modeling used by Ranjan et al. (2010), where the wire and wall are considered in contact and merged with an appropriate fillet. In the second case the wire and wall are separated by a nominal gap $g$. For the present case $L_{x}=4 \pi h, L_{z}=8 \pi h$, and $D=h$. The nominal gap is set to equal to $g=0.05 h$, in order to allow a small but significant cross-flow.

No heat transfer calculation was performed in the work by Ranjan et al. (2010). In the present simulations a heat source is specified within the walls while it is assumed that no that heat is generated in the wire.

\subsection{Mathematical Model}

The conjugate heat-transfer problem is solved by resolving the incompressible, constant-property, Navier-Stokes equation in the fluid (1-3) and the conduction equation in the solid (4):

$$
\begin{aligned}
& \frac{\partial u_{i}}{\partial x_{i}}=0 \\
& \frac{\partial u_{i}}{\partial t}+u_{j} \frac{\partial u_{i}}{\partial x_{j}}=-\frac{1}{\rho} \frac{\partial p}{\partial x_{i}}+v \frac{\partial^{2} u_{i}}{\partial x_{j} \partial x_{j}} \\
& \frac{\partial T}{\partial t}+u_{j} \frac{\partial T}{\partial x_{j}}=\alpha_{f} \frac{\partial^{2} T}{\partial x_{j} \partial x_{j}} \\
& \frac{\partial T}{\partial t}=\alpha_{s} \frac{\partial^{2} T}{\partial x_{j} \partial x_{j}}+q
\end{aligned}
$$

where $u$ is the velocity field, $T$ is the temperature, $v$ is the viscosity, $\alpha$ is the thermal diffusivity ( $s$ in the subscript refers to solid, $f$ for the fluid), $\rho$ is the density of the fluid, $p$ is the pressure, $t$ is the time, and $x$ is the Cartesian coordinate. Here $q$ is not the volumetric heat source but rather the ratio between the volumetric heat source and the thermal capacity of the solid; it can be seen as a temperature source. This source is set to a constant value in the heated solid and to zero in the wire.

Periodic boundary conditions are assumed in the streamwise and spanwise directions for the velocity, with a constant pressure gradient in both directions determined dynamically in order to maintain a constant mass-flow rate. Similar configurations are common in the simulation of parallel channel flows. In the case of conjugate heat transfer, special attention needs to be paid to the energy equations, since the temperature field is not periodic. If the temperature source distribution is constant, however, the problem can be reduced to a periodic one for the temperature as well (Iaccarino et al., 2002).

The temperature in a configuration such as the one examined here can be written as

$$
T=T_{0}+\gamma z+\widetilde{T},
$$

which represents a linear gradient in direction $\mathrm{z}$ (streamwise direction) superposed on a periodic temperature field $\widetilde{T}$. The ramp of the gradient can be determined by the conservation of energy 
principle (since the energy is evacuated from the system only through the fluid, if higher-order axial conduction is neglected (Ghaddar et al., 1986):

$\gamma=q \frac{c_{p, s}}{c_{p, f}} \frac{A_{S}}{A_{f}} \frac{1}{W_{b u l k}}$

Here, $\frac{A_{S}}{A_{f}}$ is the ratio between the area of the solid and the fluid (on a plane normal to z), and $W_{b u l k}$ is the streamwise bulk velocity. We note that $\gamma$ depends on the ratio between the heat capacity of the solid and the heat capacity of the fluid $\frac{c_{p, s}}{c_{p, f}}$. In fact, $\gamma$ is a measure of how easily the fluid is able to evacuate heat from the solid. If the heat capacity of the fluid is significantly higher than the heat capacity of the solid, the same temperature source in the solid will cause a smaller, streamwise fluid temperature increase. A high value of $\gamma$ indicates a poor coolant, whereas a low value indicates an effective coolant.

Substituting (5) into (3) and (4) leads to the following set of equations for the temperature:

$$
\begin{aligned}
& \frac{\partial \widetilde{T}}{\partial t}+u_{j} \frac{\partial \widetilde{T}}{\partial x_{j}}=\alpha_{f} \frac{\partial^{2} \widetilde{T}}{\partial x_{j} \partial x_{j}}-u_{3} \gamma \\
& \frac{\partial \widetilde{T}}{\partial t}=\alpha_{s} \frac{\partial^{2} \widetilde{T}}{\partial x_{j} \partial x_{j}}+q .
\end{aligned}
$$

The equations are balanced in the sense that the energy produced in the solid is dissipated in the fluid. No energy is transferred at the boundaries. Therefore, periodic boundary conditions can be applied for the temperature field $\widetilde{T}$.

\subsection{Choice of Parameters}

The relevant parameters for the following problem are the Reynolds numbers in direction $\mathrm{x}$ and $\mathrm{z}$; the Prandtl number; the ratio between the thermal diffusivity in the solid and the thermal diffusivity in the liquid; and, to a minor extent, the ratio between the thermal capacities. The Reynolds number in direction $\mathrm{z}$ can be defined as

$\operatorname{Re}_{z}=\frac{W_{b u l k} h}{v}$,

which is chosen to be equal to 6,000 (consistently with Ranjan et al. (2010), albeit slightly higher). $W_{\text {bulk }}$ is the bulk velocity in the streamwise direction. The Reynolds number in direction $\mathrm{x}$ can be defined as

$$
\operatorname{Re}_{x}=\frac{U_{b u l k} h}{v}
$$

which is chosen to be equal to 1,000 (consistently with Case C in Ranjan et al. (2010), albeit slightly higher). $U_{b u l k}$ is the bulk velocity in the spanwise direction. The Prandtl number can be defined as 
$\operatorname{Pr}=\frac{v}{\alpha_{f}}$

which is chosen to be equal to 0.01 , a value representative of a liquid metal coolant. The ratio between the two thermal diffusivities is assumed to be equal to 10 (the thermal diffusivity in the liquid being 10 times higher than the solid), and the ratio between the thermal capacities is assumed to be equal to 1 . This corresponds loosely to the case of a liquid metal coolant and a uniform metallic fuel.

We note that the source term does not appear as a relevant parameter of choice. The reason is the following. There exists a normalization of the temperature $\widetilde{T}$ (and notably $\gamma L$, where $L$ is a lenghtscale of arbitrary choice) for which the source term can be removed from the equation. Since $\widetilde{T}$ is a fluctuation on the linear streamwise gradient, it is intuitive that the choice of $q$ is irrelevant for its determination. An increased value of $q$ would have a significant effect on $\gamma$, however, thereby influencing the distribution of $T$.

On the upper and lower boundaries for the solid, a zero heat flux condition is applied (the temperature derivative is set to zero on the boundary).

\subsection{Numerical Methods}
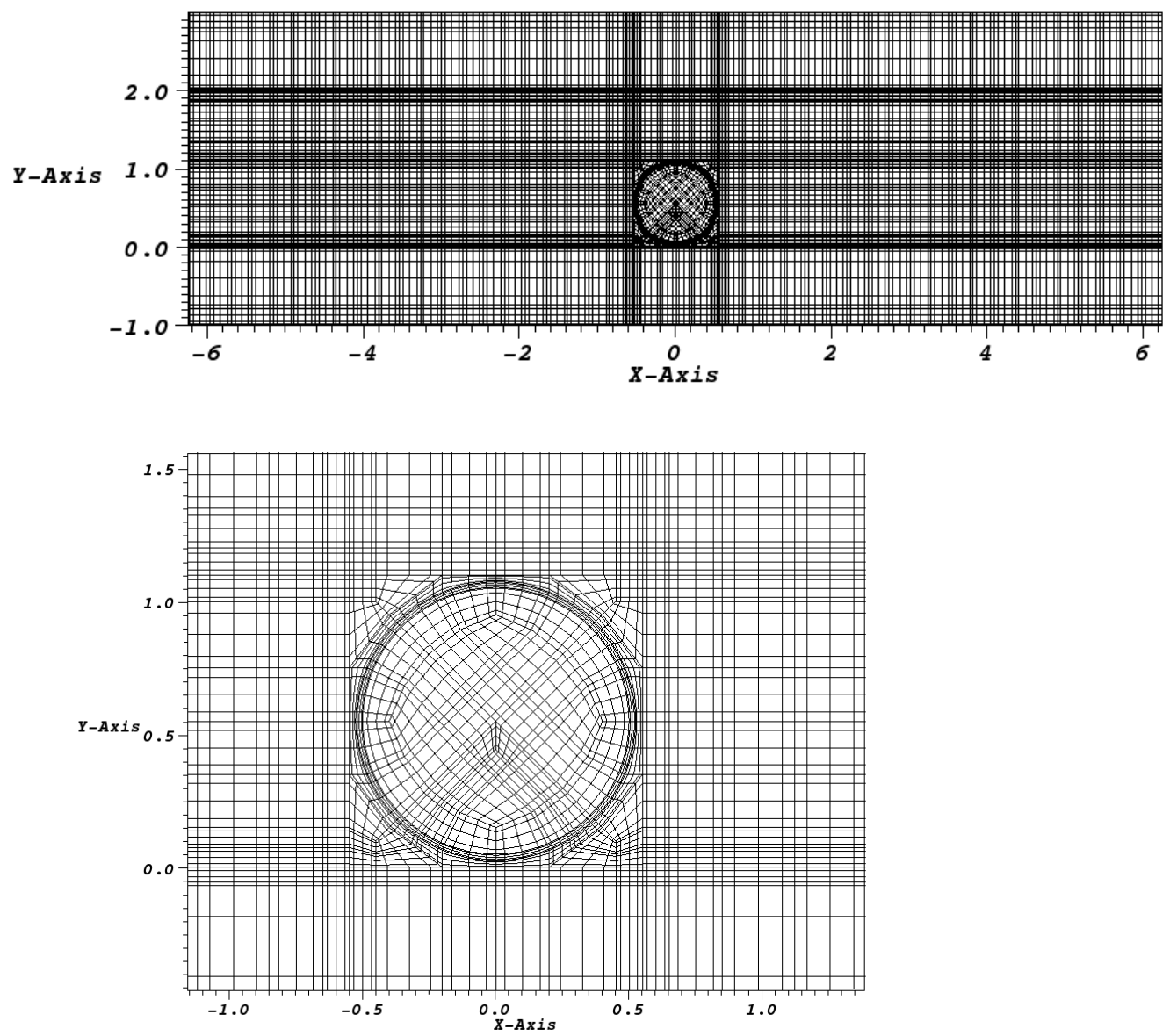

Fig. 2. Grid structure: (top) very coarse grid (fourth-order polynomial) for the no-contact case; (bottom) detail. 
In this subsection we present the methodology used to simulate the flow field. The simulations were carried out by using the spectral element code Nek5000 in LES mode on two Argonne computer systems - Cosmea (a 32-node, 128-core Linux cluster) and Blue Gene/P (an IBM system with 40,960 quad-core compute nodes) - with up to 15,000,000 collocation points. An example of structure of the macroscopic grid used is shown in Fig. 2 for the streamwise-normal cross section; the figure depicts the elements and the actual collocation points for a very coarse mesh with no contact between wire and wall. The discretization is designed to allow for at least one point near the wall at $\mathrm{y}^{+}<1$ and five points within $\mathrm{y}^{+}<10$.

Spectral elements methods are a class of higher-order methods in space. Polynomial functions of up to the $11^{\text {th }}$ degree have been used to discretize the velocity field in each element. In the generalized weighted residual framework, the present spectral element method can be classified as a Galerkin method where the test functions and the basis functions for each element are Lagrange polynomials evaluated on Gauss-Lobatto-Legendre collocations points (for the velocity).

In LES, large-scale turbulence is simulated while smaller scales are modeled. Since smaller scales have a nearly universal behavior, LES is a more reliable methodology than is Reynolds-averaged Navier-Stokes (RANS), in the sense that it generally depends less on the model details. It is also preferable to use direct numerical simulation because it can be performed at a reasonable computational cost. The contribution of the smaller scales to the energy cascade is in the present case modeled through a local, element-based explicit cutoff filter in wave-number space. The energy is removed from the smallest simulated scales (high wave-numbers), thus mimicking the effect of smaller eddies (Fischer and Mullen, 2001). This procedure allows for a coarser grid and therefore lower computational cost. Time advancement has been carried out through an explicit third-order, backward finite-difference scheme. An additional benefit of this method is that it does not explicitly define an eddy viscosity, leaving the definition of a turbulent Prandtl number as superfluous. The Nek5000 code has been extensively validated and is massively parallel. In the present case the time stepping was chosen to have a maximum Courant number no higher than 0.4. For comparison, some calculations were also run by using the industrial-strength code STAR-CCM+ in the RANS framework with a realizable k- $\varepsilon$ turbulence model and all- $\mathrm{y}^{+}$wall modeling.

\section{RESULTS AND DISCUSSION}

Several cases were run corresponding to two different geometries and different mesh resolutions. While the macroscopic mesh was not changed during the simulations, mesh convergence was proven by increasing the order of the polynomial basis from the $4^{\text {th }}$ order up to the $11^{\text {th }}$.

\subsection{Velocity Distribution}

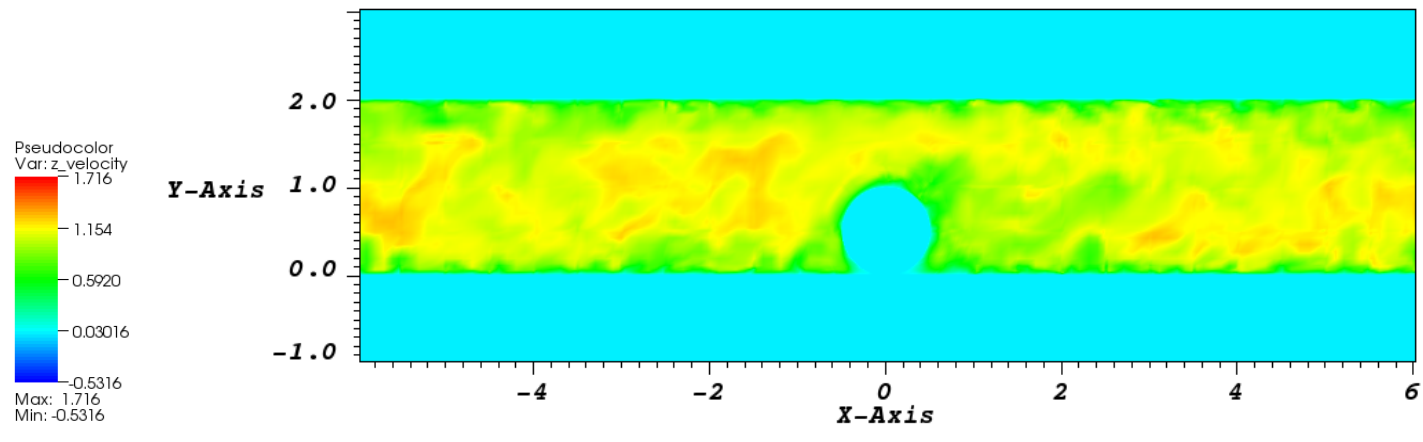

Fig. 3. Instantaneous streamwise velocity in a z-normal plane. Spatial units are normalized by h. 
We begin by comparing the velocity field with the data of Ranjan et al. (2010). Figure 3 shows an instantaneous contour plot for the streamwise velocity, while Figure 4 shows a contour plot for the averaged streamwise velocity; both figures refer to the case with contact between wire and wall. Compared with similar plots published by Ranjan et al. (2010), these figures show a good degree of accuracy considering that the Reynolds number is $10 \%$ higher than that for the DNS case.

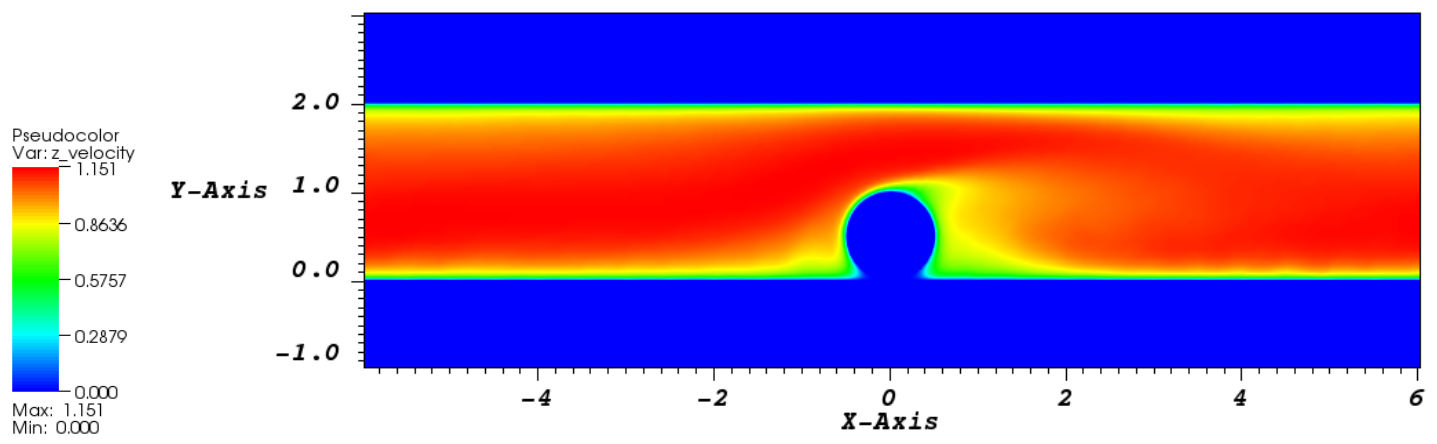

Fig. 4. Time-averaged streamwise velocity in a z-normal plane. Spatial units are normalized by $h$.
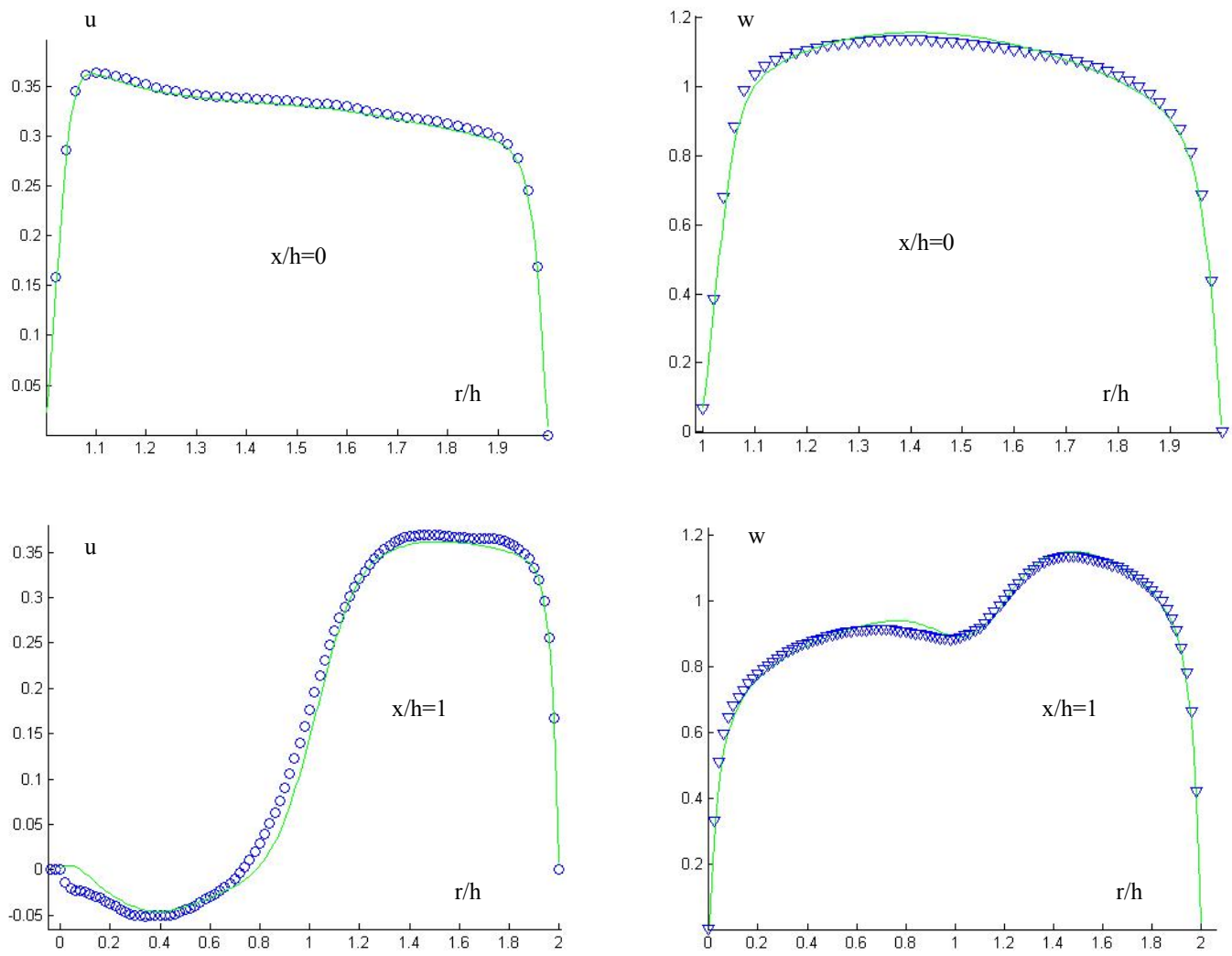

Fig. 5. Comparisons of the streamwise velocity and the cross velocity at $\mathrm{x} / \mathrm{h}=0.0$ (wire centerline) and $\mathrm{x} / \mathrm{h}=1.0$. The solid line represents the DNS data. All values are normalized by $\mathrm{W}_{\text {bulk. }} . \mathrm{r}$ represents the distance from the bottom wall. 
Figure 5 shows four profile comparisons of the results obtained with Nek5000 (with contact modeling) and the DNS data. The accuracy is good considering the enormous difference in resolutions (we used less than one-tenth of the collocation points) and the difference in Reynolds number. All profiles refer to time-averaged data. (For the position in $\mathrm{x}$, see Fig. 3.)

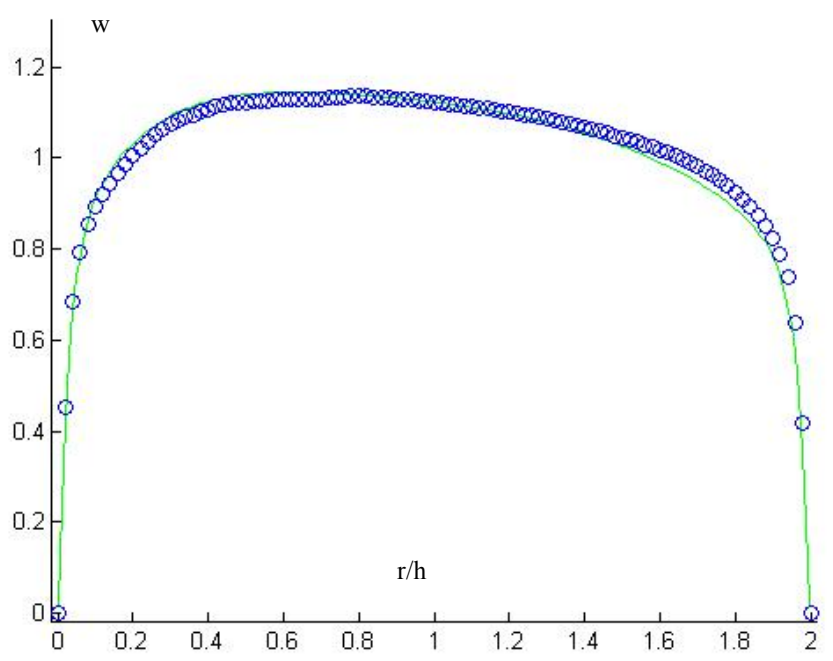

Fig. 6. Comparisons of the streamwise velocity at $\mathrm{x} / \mathrm{h}=6$. Values are normalized by $\mathrm{W}_{\text {bulk. }}$. The solid line represents the DNS data; $r$ represents the distance from the bottom wall.

Figure 6 shows a comparison between the Nek5000 results for the case with no contact between wire and wall and the DNS data. Such a comparison would be meaningful, for validation purposes, only far from the wire. We therefore did the comparison at $\mathrm{x} / \mathrm{h}=6.0$ : the agreement seems reasonable and within the scope of the present work. Time-averaging convergence was verified.

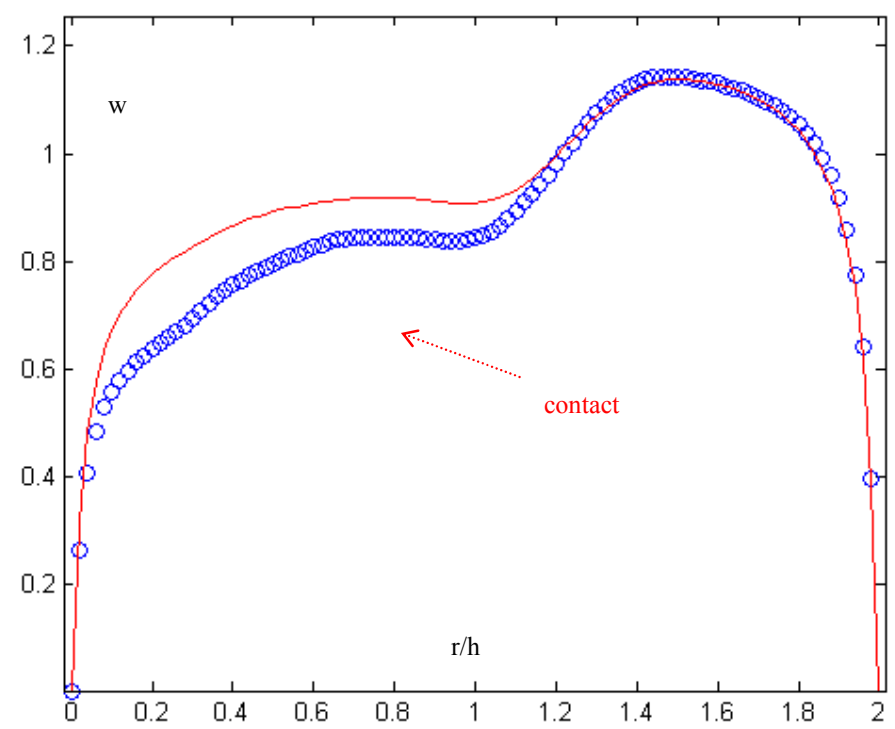

Fig. 7. Comparison of the streamwise velocity between the two Nek5000 cases at $\mathrm{x} / \mathrm{h}=6$. Values are normalized by $\mathrm{W}_{\text {bulk }}$; $\mathrm{r}$ represents the distance from the bottom wall. The solid line represents the case with contact between wall and wire. 
Near the wire, the two cases (i.e., no contact and contact between wire and wall) behave differently. In the case of no contact, the velocity distribution appears to be altered. Figure 7 compares the two Nek5000 results at $\mathrm{x} / \mathrm{h}=1.0$. Near the bottom wall $(\mathrm{r} / \mathrm{h}=0.0$, where $\mathrm{r}$ is the distance from the bottom wall), the streamwise velocity is lower, an effect that could be related to the presence of a higher cross-flow in this area.

In fact, as it can be seen in Fig. 8, the recirculation pattern near the gap is altered when compared to the case with no contact. Moreover the cross velocity in the narrow gap is significantly higher than the surrounding areas, probably because of local acceleration. This effect is nontrivial, and we plan to investigate it in further detail. It is also relevant from the point of view of the hydrodynamics.

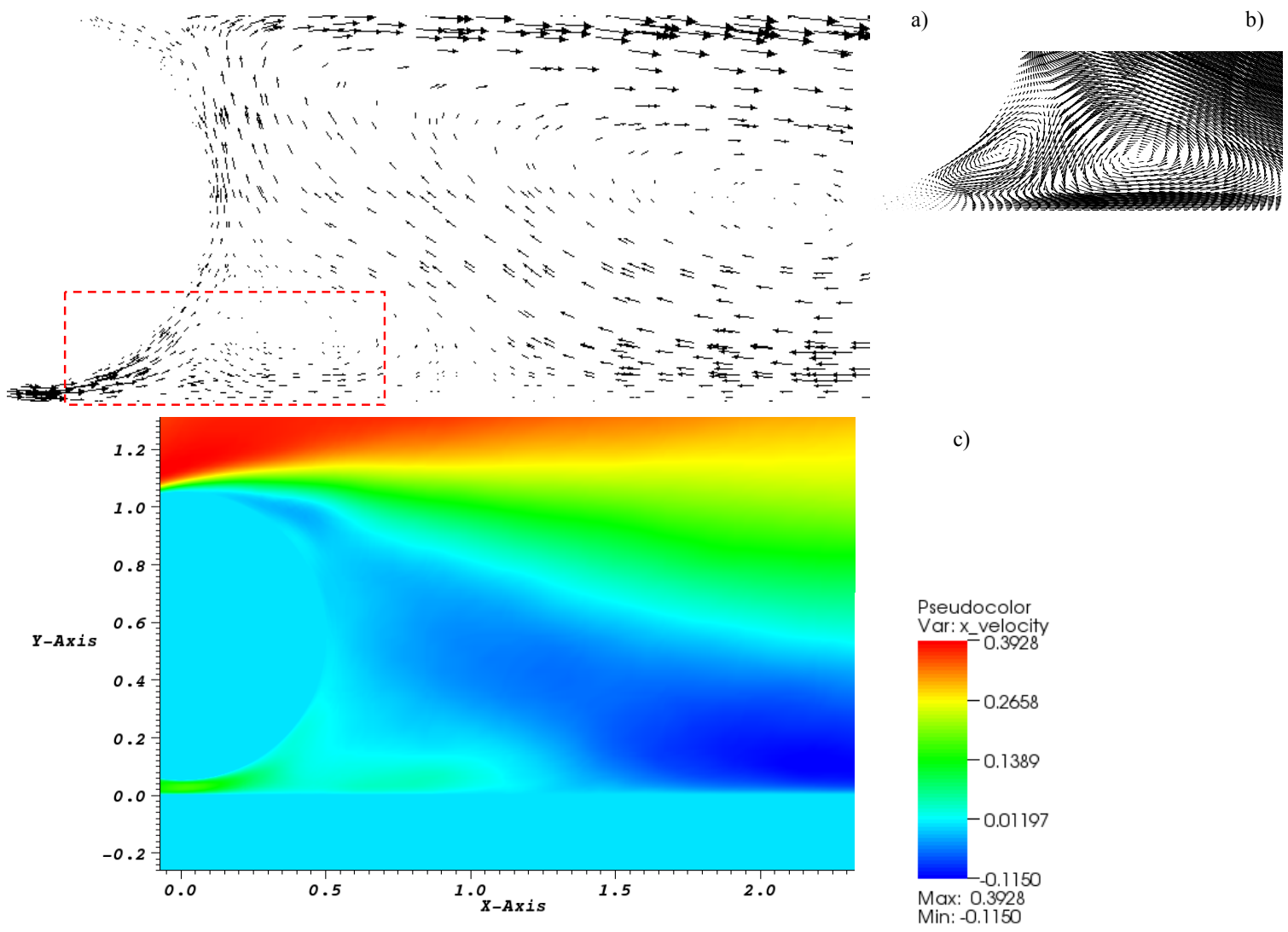

Fig. 8. Cross velocity: (a) vector plot for the case with no contact; (b) near-gap vector plot for the case with contact; (c) contour plot of the velocity in direction $\mathrm{x}$ for the case with no contact.

\subsection{Temperature}

Figure 9 shows the average temperature distribution for the case with contact between wire and wall. The plots in Figs. 9 and 10 clearly are similar except for the wire region (and the statistical noise still present in Fig. 10). A hot spot can be observed in both cases near $\mathrm{x}=0$ and $\mathrm{r}=-\mathrm{h}$ (near the lower adiabatic boundary), with the hot spot in the no-contact case shifted toward $x>0$. Note that the scales are different. It is evident however that in the case with contact between wire and pin the hot spot is slightly higher. 


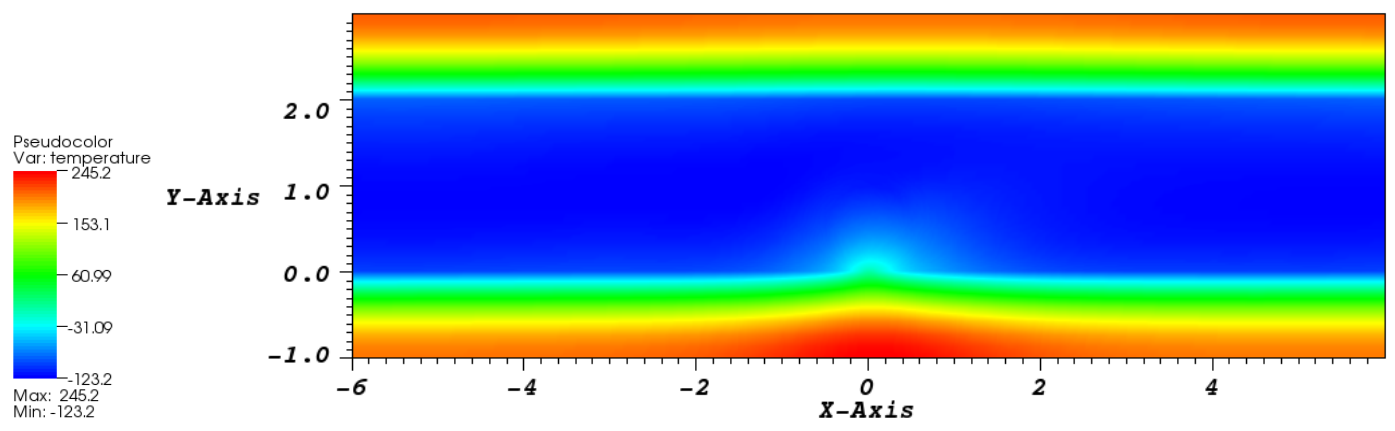

Fig. 9. Average temperature $\widetilde{T}$ distribution for the case with contact.

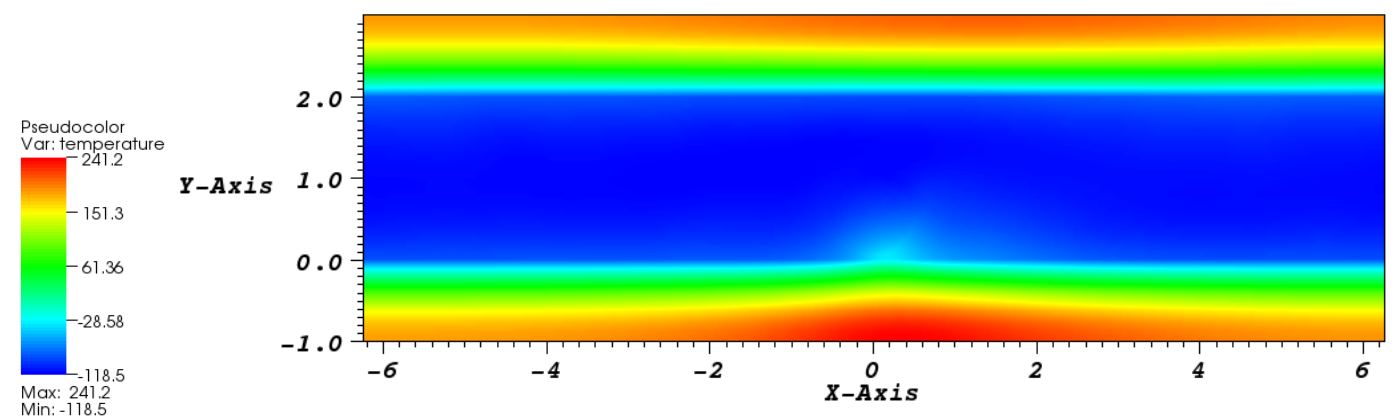

Fig. 10. Average temperature $\widetilde{T}$ distribution for the case with no contact.

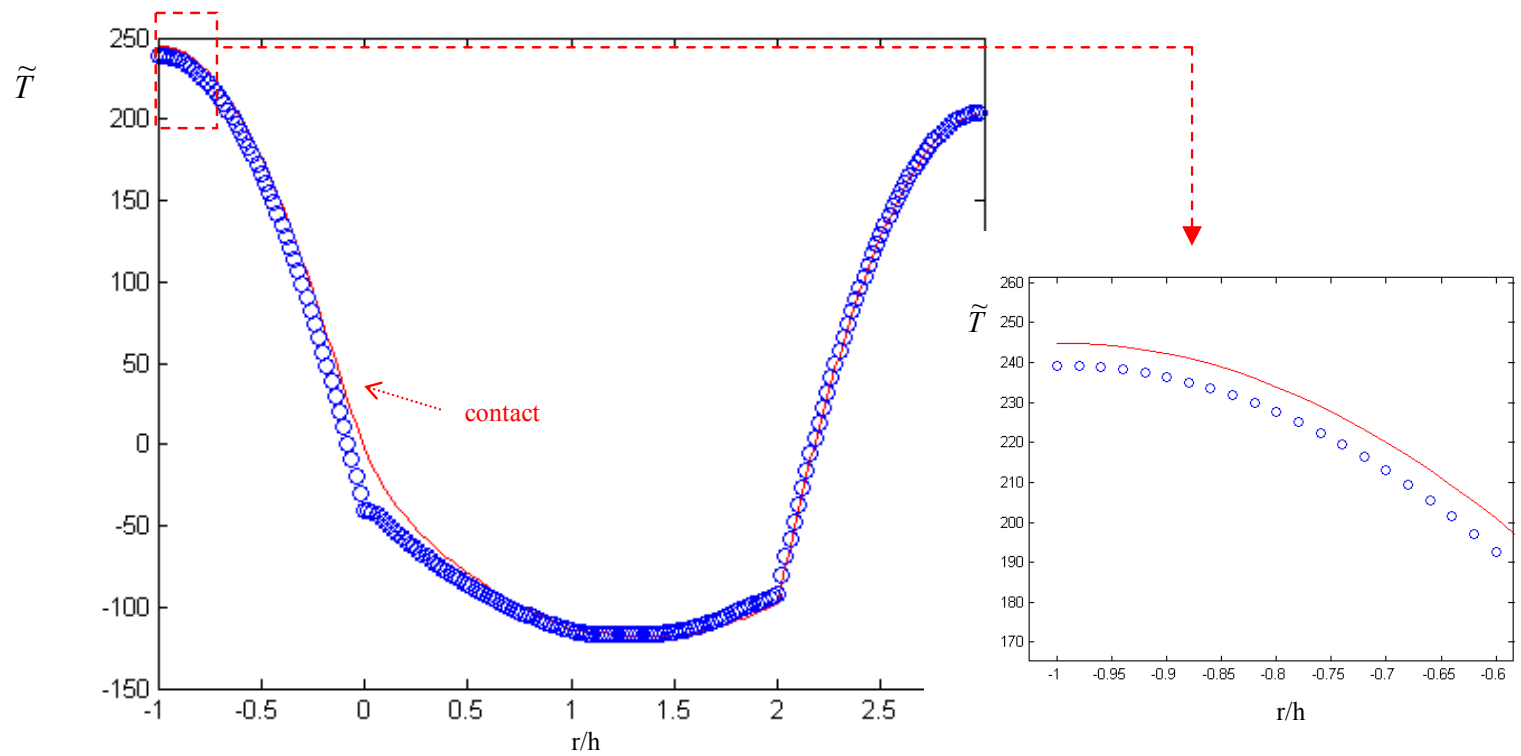

Fig. 11. Temperature $\widetilde{T}$ profile at $\mathrm{x} / \mathrm{h}=0.0$ for the two cases. Detail of the distribution near the lower adiabatic boundary is shown in the right box. The solid line represents the case with contact between wall and wire. 
To provide a more quantitative comparison, we are also reporting the temperature profiles for $\mathrm{x} / \mathrm{h}=0$ in Fig. 11. While the two profiles are different and the case with contact clearly predicts a higher hot spot, the difference appears to be small. We conclude that the significant difference observed in the hydrodynamic side had little effect on the hot-spot prediction.

We note, however, that the temperatures of the fluid at the wall near the contact region between wire and pin is significantly affected by the contact modeling. In the case with no contact the peak $\widetilde{T}$ in the fluid was evaluated to be -31.5 , while in the case with contact it was evaluated to be -4.77 . This is a significant temperature increase (about $10 \%$ of the maximum temperature difference within the model). The peak fluid temperature is an important design parameter because it determines the margin to boiling.

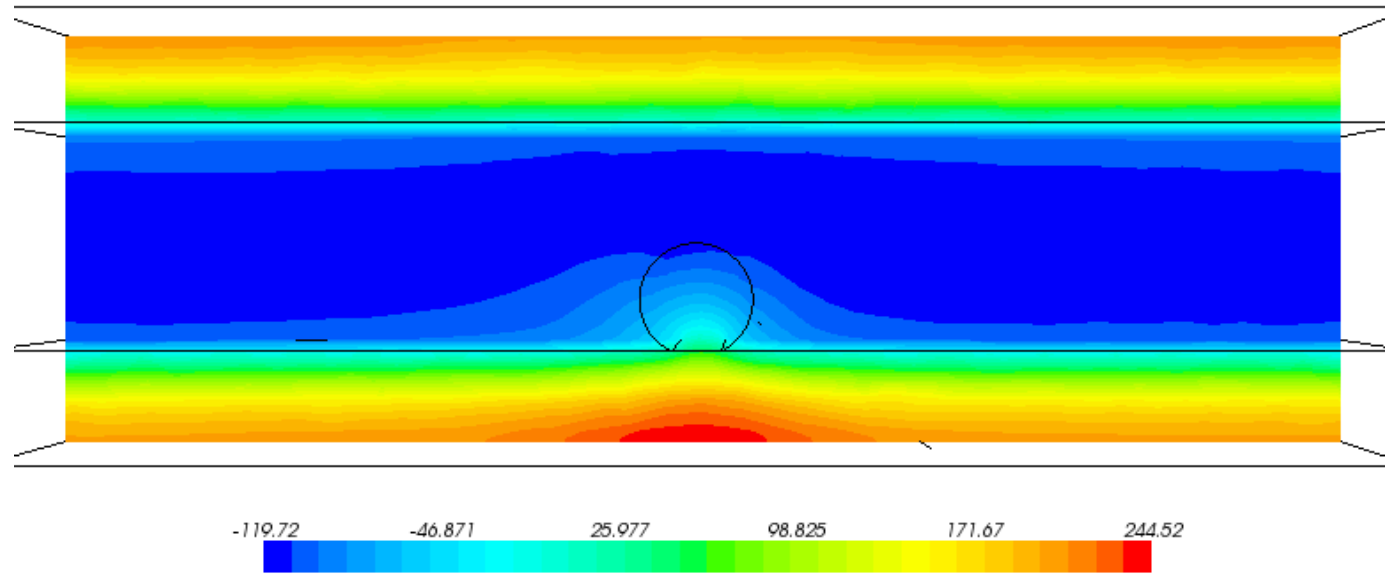

Fig. 12. Temperature $\widetilde{T}$ for the case with contact, obtained by using STAR-CCM+.

Figures 12 and 13 provide further verification of the results obtained for the temperature. Figure 12 shows the average temperature for the STAR-CCM+ case with contact between wall and wire. Figure 13 shows a comparison between the temperature profile obtained by Nek5000 and that by STARCCM+ for the case with contact between wire and wall. The results are in very good agreement. For the present case RANS appears sufficiently accurate to predict the value of the hot spot.

\subsection{Summary}

The results presented here suggest that the modeling of the contact between wire and wall is significant, if not crucial, from the hydrodynamic point of view. It is less so from the point of view of the hot-spot prediction, since a significant difference in hydrodynamic behavior leads to only a few percentage points difference in the value of the hot spot. The peak temperature of the fluid, on the other hand, appears to be significantly affected, thus warranting further investigation of this issue.

An additional conclusion that can be drawn is that RANS-based techniques are sufficient to reach accurate results for the prediction of the hot spot, for the present geometry and parameters. This seems to be due to the conduction-dominated nature of heat transfer for low-Prandtl fluid conjugate heat transfer cases. 


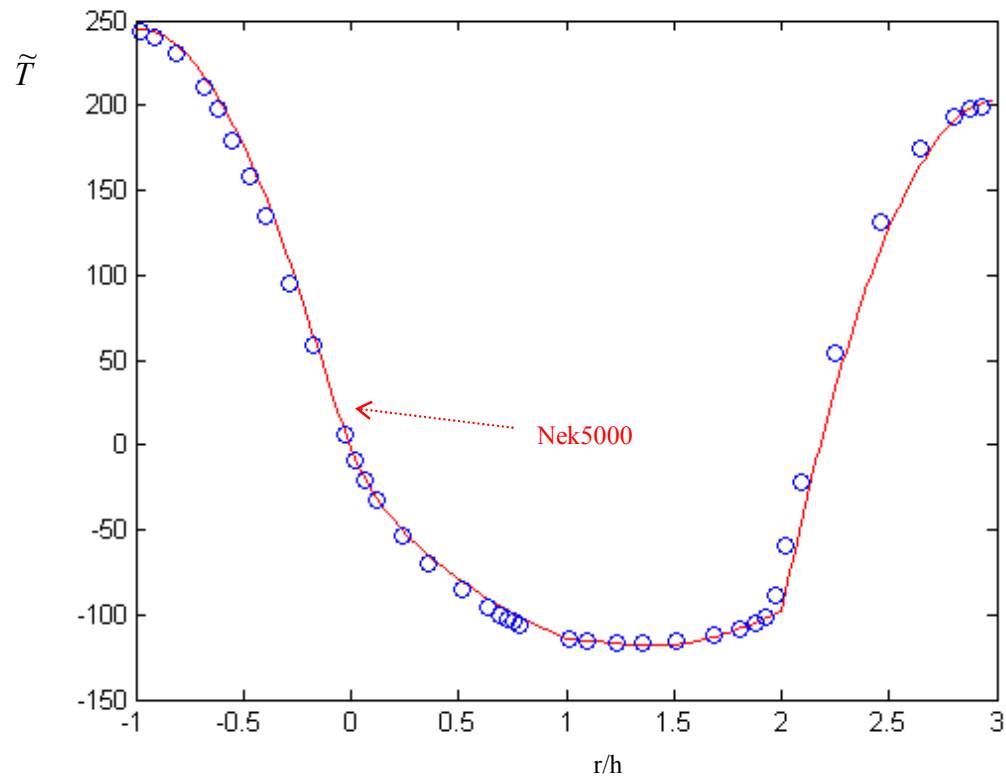

Fig. 13. Temperature $\widetilde{T}$ profile at $\mathrm{x} / \mathrm{h}=0.0$ for the case with contact, obtained by using Nek5000 and STAR-CCM+. The solid line represents the case performed using Nek5000.

\section{ACKNOWLEDGMENTS}

This work was supported in part by the Office of Advanced Scientific Computing Research, Office of Science, U.S. Department of Energy, under Contract DE-AC02-06CH11357.

\section{REFERENCES}

Baglietto, E., and N. Hisashi, "Improved turbulence modeling for performance evaluation of novel fuel designs," Nuclear Tech., 158, pp. 237-248 (2007)

Fischer, P.F., J. Lottes, W.D. Pointer, and A. Siegel, "Petascale algorithms for reactor hydrodynamics," J. Phys. Conf. Series, 125, 012076 (2008)

Fischer, P.F., and J.S. Mullen, "Filter-based stabilization of spectral element methods," Comptes Rendus de l'Académie des sciences Paris, 332, Série I - Analyse numérique, 265-270 (2001)

Ghaddar, N.K., G.E. Karniadakis, and A.T. Patera, "A conservative isoparametric spectral element method for forced convection; application to fully developed flow in periodic geometries," Numerical Heat Transfer, Part B: Fundamentals, 9, issue 3, pp. 277-300 (1986)

Iaccarino, G., A. Ooi, P.A. Durbin, and M. Behnia, "Conjugate heat transfer predictions in twodimensional ribbed passages," Intl. J. Heat and Fluid Flow, 23, pp. 340-345 (2002)

Khakim, A., E. Merzari, and H. Ninokata, "Feasibility study of the application of exotic pin for tightlattice fuel assembly," Trans. Amer. Nuclear Soc., 100, p. 747 (2009)

Pointer, W.D., J. G. Smith, P. Fischer, and A. Siegel, "RANS simulations of turbulent diffusion in wire-wrapped sodium fast reactor fuel assemblies," abstract in International Conference on Fast Reactors and Related Fuel Cycles, Kyoto, pp. 249-250, December 2009 
$\mathrm{Qu}, \mathrm{W}$. , and Issam Mudawar, "Analysis of three-dimensional heat transfer in micro-channel heat sinks", International Journal of Heat and Mass Transfer, 45, pp. 3973-3985 (2002)

Ranjan, R., C. Pantano, and P. Fischer, "Direct simulation of turbulent swept flow over a wire in a channel," J. Fluid Mechanics, 651, pp. 165-209 (2010)

Tang, Y.S., R.D. Coffield Jr., and R.A. Markley Jr., Thermal Analysis of Liquid-Metal Fast Breeder Reactors, American Nuclear Society (1978) ISBN: 0-89448-011-1

The submitted manuscript has been created by UChicago Argonne, LLC, Operator of Argonne National Laboratory ("Argonne"). Argonne, a U.S. Department of Energy Office of Science laboratory, is operated under Contract No. DE-AC02-06CH11357. The U.S. Government retains for itself, and others acting on its behalf, a paid-up nonexclusive, irrevocable worldwide license in said article to reproduce, prepare derivative works, distribute copies to the public, and perform publicly and display publicly, by or on behalf of the Government. 